\title{
Carrier frequency
}

National Cancer Institute ( $\mathrm{NCl})$

\section{Source}

National Cancer Institute (NCI). Carrier frequency.

The proportion of individuals in a population who have a single copy of a specific recessive gene mutation; also sometimes applied to the prevalence of mutations in dominantly acting genes such as BRCA1 and BRCA2. Also called carrier rate. 